\title{
Research Article \\ Effect of Chamfer Angle on the Calibration Curves of Five Hole Probes
}

\author{
Nekkanti Sitaram and Kancherla Srikanth \\ Thermal Turbomachines Laboratory, Department of Mechanical Engineering, IIT Madras, Chennai 600 036, India \\ Correspondence should be addressed to Nekkanti Sitaram; nsitaram.iitm@gmail.com
}

Received 30 May 2014; Accepted 28 August 2014; Published 15 September 2014

Academic Editor: Takayuki Matsunuma

Copyright (C) 2014 N. Sitaram and K. Srikanth. This is an open access article distributed under the Creative Commons Attribution License, which permits unrestricted use, distribution, and reproduction in any medium, provided the original work is properly cited.

\begin{abstract}
Five hole probes are extensively used for measurement of total and static pressures, flow angles, velocity and its components in turbomachinery, and other aerodynamic flows. Their operating range is usually limited to $30-40^{\circ}$ depending on the type of the probe head. The chamfer angle of the probe is usually taken around $45^{\circ}$. Recent studies on three hole probes have shown that $30^{\circ}$ chamfer angle is desirable for unsteady flow measurements. Hence the present investigation is undertaken to find the optimum chamfer angle of five-hole probes. A special five-hole probe of $9.6 \mathrm{~mm}$ head diameter and $3 \mathrm{~mm}$ diameter pressure take off tubes was designed and fabricated. The large size of the probe was chosen to minimize machining inaccuracies. The probe chamfer angle was varied from $30^{\circ}$ to $60^{\circ}$ in $5^{\circ}$ steps. For each of the chamfer angles, the probe was calibrated in the range of $-30^{\circ}$ to $+30^{\circ}$ in $5^{\circ}$ interval and the calibration curves are presented. In addition the sensitivities of the calibration coefficients are determined. It is concluded that five-hole probe with a chamfer angle $30^{\circ}$ has large operating range, while five-hole probe with a chamfer angle of $50^{\circ}$ has good sensitivity.
\end{abstract}

\section{Introduction}

In general flow can be analysed by three techniques, namely, flow visualization, computational methods, and measurements of flow parameters. Computational methods are expensive to develop and use. Flow visualization techniques serve only to locate flow regions of interest. Obtaining quantitative data often requires direct measurement of the flow. One such method of direct measurement is by inserting multihole pressure probes into the flow.

Multihole pressure probes have been conveniently used to determine static and total pressures and flow angles in two mutually perpendicular planes (named yaw and pitch planes) in three-dimensional flow fields with suitable calibrations. From these four flow parameters, flow velocity and its three components can be determined. Multihole pressure probes thus combine the means for simultaneous measurement of total, static, and dynamic pressures and flow directions with one instrument. When designing a pneumatic probe that is to be used for flow measurements, the effects of blockage, frequency response, pressure hole size and geometry, the local
Mach and Reynolds numbers, and the relative scale of the phenomenon under investigation must be specified.

For measuring three-dimensional flows, multihole probes with four, five, seven, or even higher number of pressure holes strategically placed on aerodynamic bodies such as sphere, hemisphere, and prism can be used. In principle a four-hole probe can measure the four quantities that are required to completely define the flow. However for the sake of symmetry in both yaw and pitch planes, five hole probes are usually employed. When the yaw and pitch angles of the flow exceed the usual operating range of five hole probes, seven hole probes or probes with larger number of holes are employed.

Five hole probes Treaster and Yocum [1] are extensively used for measurement of total and static pressures, flow angles, velocity and its three components in highly complex and three-dimensional turbomachinery, and other aerodynamic probes. Five hole probes also find applications in other flows (automotive wheel arch flow, Malviya et al. [2], supersonic flow measurements, Naughton et al. [3] and Milanovic and Kalkhoran [4]). Five hole probes also find novel applications, such as measurement of skin friction in 

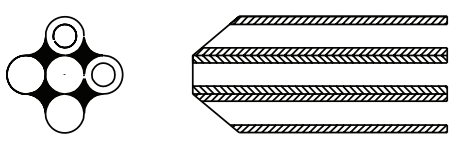

(a) Forward facing tubes

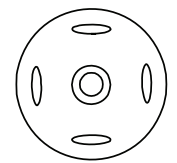

(d) Cone head with perpendicular holes
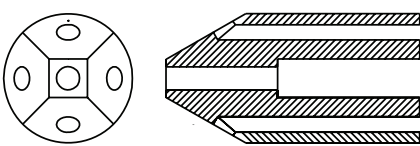

(b) Pyramid head with perpendicular holes

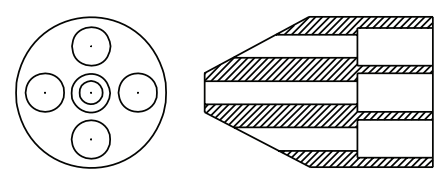

(c) Cone head with forward facing holes
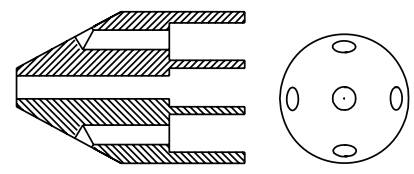

(e) Hemispherical head with forward facing holes
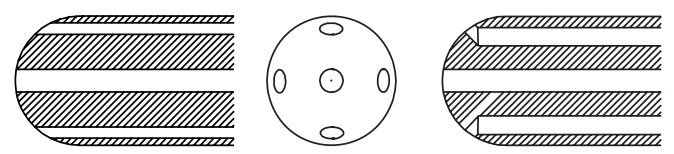

(f) Hemispherical head with perpendicular holes

FIgURE 1: Geometry of five-hole probe heads Dominy and Hodson [10].

complex two- and three-dimensional flows Lien and Ahmed [5]. Recently Telionis et al. [6] had made a comprehensive survey of multihole pressure probes for flow measurements.

The shape of the head of the five-hole probe can vary widely as shown in Figure 1. Probes with forward facing tubes are usually inexpensive and can be fabricated in miniature size (about $1.22 \mathrm{~mm}$ diameter tip, Ligrani et al. [7]). However their operating angle is usually limited to $30^{\circ}$, while the operating range of probes with perpendicular holes is limited to $40^{\circ}$ Pisasale and Ahmed [8]. The limitation is due to the value of denominator, $D$, becoming very small, zero or negative, when yaw or pitch angle exceeds a certain value. In such case, the calibration coefficients become very large or singular or changes sign. The chamfer angle of the probe is usually taken as $45^{\circ}$ (or in the range of $40-50^{\circ}$ ). But recent studies on three hole probes Díaz et al. [9] have shown that $30^{\circ}$ chamfer angle is desirable for unsteady flow measurements. Hence the present investigation is undertaken with the objective of determining the effect of chamfer angle on the calibration curves of five hole probes to find the optimum chamfer angle. To the best of the knowledge of the authors, no such systematic investigations are undertaken. Dominy and Hodson [10] had calibrated three conical probes with perpendicular holes. The cone angles of the probes were 45 , 60 , and $90 \mathrm{deg}$, respectively, corresponding to chamfer angles of $67.5,60$, and $45 \mathrm{deg}$. However they had not examined the effect of chamfer angle on the calibration coefficients and their sensitivity.

\section{Design and Fabrication of Five-Hole Probe}

2.1. Five-Hole Probe. A $9.6 \mathrm{~mm}$ diameter five-hole probe was fabricated with the tip of the probe shaped as truncated cone. The probe was made of a $9.6 \mathrm{~mm}$ brass rod with five holes of $3 \mathrm{~mm}$ diameter drilled in a + format with a clearance of $0.15 \mathrm{~mm}$ between the holes and outer circumference. Five $3 \mathrm{~mm}$ diameter stainless steel tubes were fitted tightly into these holes. The diameter of the tubes was reduced slightly by grinding, so that the tubes would fit into the holes. The tubes were silver brazed to the brass body at the rear end with $10-15 \mathrm{~mm}$ of the tubes exposed. Plastic tubes were fitted to the exposed ends of the tubes. Among the five tubes, one forward-facing tube was at the centre, two chamfered side tubes were on the horizontal axis, and remaining two side tubes were on the vertical axis as shown in Figure 2. The tubes and the brass body were integral and machined together to change the chamfer angle of the probe. The design deviated from the usual design of integral probe head and stem to facilitate easy machining of the probe head to the required chamfer angle.

2.2. Probe Holder and Support. The probe support was made up of stainless steel (SS) material. The probe support consisted of a $9.6 \mathrm{~mm}$ diameter. SS tube of $400 \mathrm{~cm}$ long is attached to a $12.7 \mathrm{~mm}$ hexagonal rod. A hole of $9.6 \mathrm{~mm}$ diameter was centrally drilled in the hexagonal rod. A small tube was perpendicularly silver brazed to the hexagonal rod. A M3 tapped hole was drilled in the center of the small tube to position the probe and to hold the probe tightly. The probe holder and support are shown in Figure 3.

\section{Calibration Tunnel and Calibration Device}

3.1. Calibration Tunnel. The probe was calibrated in the calibration tunnel available in Thermal Turbomachines Laboratory of Department of Mechanical Engineering. The probe was fixed so that its head is at the center of calibration section to minimise boundary layer and duct wall effects. A photo of the calibration tunnel is shown in Figure 4.

3.2. Calibration Device. The calibration device was mounted on the calibration section of the calibration tunnel. The probe was mounted in the central hole of the calibration device with the probe tip at the centre of the axis of the calibration tunnel. The calibration device has provisions to change the yaw angle in the range of $\pm 180 \mathrm{deg}$. at an interval of $1 \mathrm{deg}$. and pitch angle of the probe in the range of $\pm 30 \mathrm{deg}$. at an interval of $1 \mathrm{deg}$. The probe can be rotated in both clockwise and anticlockwise directions to change the yaw angle with the help of a rotating mechanism fitted onto the calibration device. It is desirable to carry out the calibrations in the yaw and pitch angle range of $-60^{\circ}$ to $+60^{\circ}$. However the pitch angle can be varied only in the range of $-30^{\circ}$ to $+30^{\circ}$. Hence the probe was calibrated at a velocity of $25 \mathrm{~m} / \mathrm{s}$ in yaw and pitch angle range of $-30^{\circ}$ to 


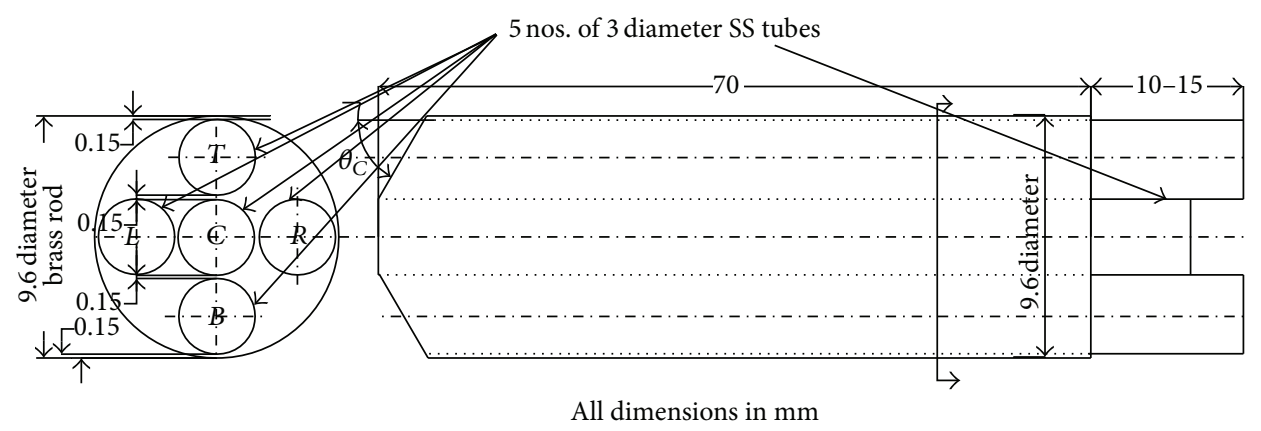

FIgUre 2: AutoCAD drawing of Five-hole probe.

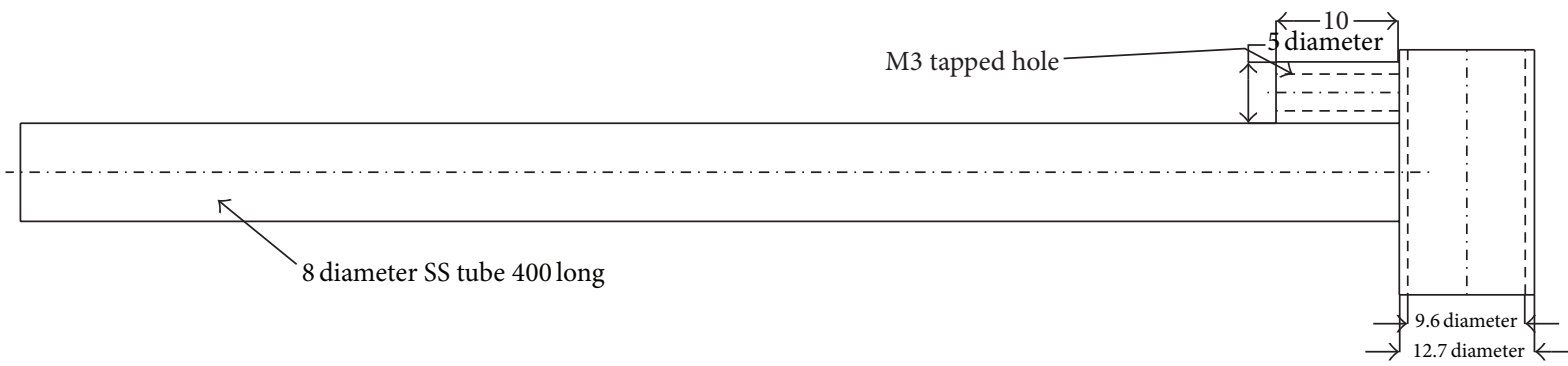

All dimensions in $\mathrm{mm}$

Figure 3: Probe holder and support.

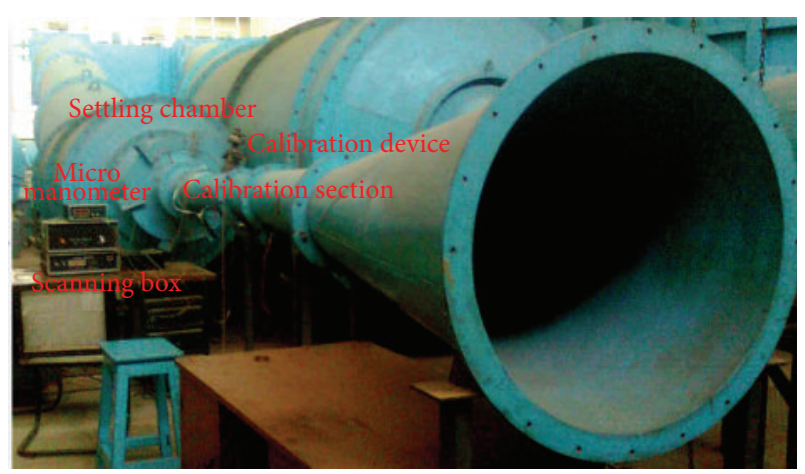

FIGURE 4: Calibration tunnel.

$+30^{\circ}$ at an interval of $5^{\circ}$. The calibration device is shown in Figure 5.

3.3. Instrumentation. The twenty-channel single selection scanning box (model no. FCO 91-3) and FC012 digital micro manometer manufactured by Furness control Ltd., Bexhill, London were used to measure probe pressure. The scanning box has twenty channels, which were numbered sequentially. The pressures to be measured were connected to the numbered inputs. The outlet channel was connected to the micromanometer. A particular channel was selected manually in the scanning box and its corresponding pressure was read from the micromanometer. The micromanometer used has a resolution of $0.1 \mathrm{~mm}$ with a range of $\pm 200 \mathrm{~mm}$

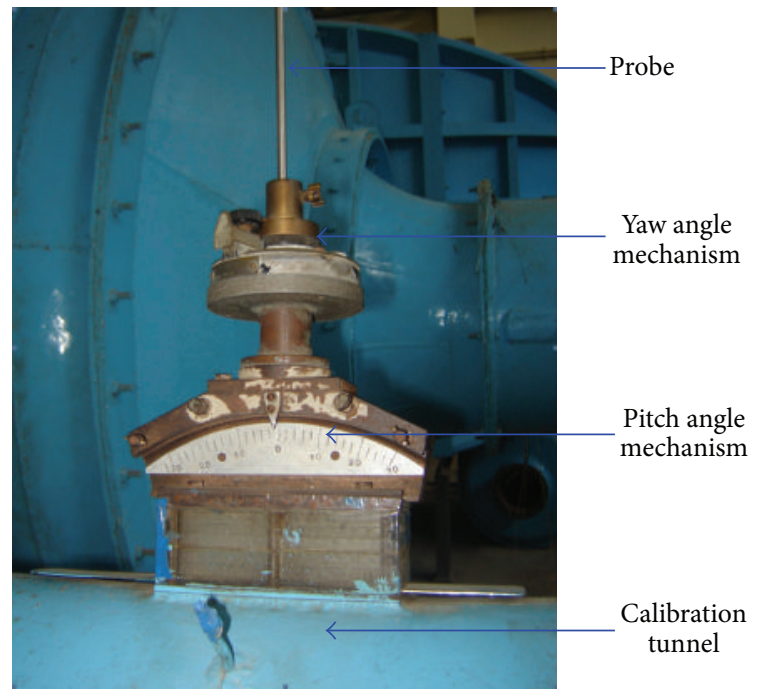

FIGURE 5: Calibration device.

of water gauge. The accuracy of the micromanometer is $\pm 0.1 \mathrm{~mm}$ of the water column. The output of the scanning box was connected to the micromanometer and it gave reading directly in terms of velocity in $\mathrm{m} / \mathrm{s}$ or pressure in $\mathrm{mm}$ of water gauge. Time constant potentiometer was used to get time averaged pressures. 


\section{Experimental Procedure and Programme}

4.1. Experimental Procedure. The calibration of the five-hole probe for the present experimental investigation was carried out in the low-speed calibration tunnel. Free stream velocity of air was maintained at $25 \mathrm{~m} / \mathrm{s}$ determined from the settling chamber pressure and calibration section wall static pressure. The five pressure tubes of the probe along with the settling chamber wall static pressure and calibration section wall static pressure taps were connected to a scanning box, which enabled to measure multipressures using just one pressure measuring instrument (digital micromanometer in this case). The fluctuating pressure signals were typically averaged over a period of 5 seconds time to allow for conditions to reach steady-state. Using the calibration device, the pitch and yaw angles of the probe were changed by $5^{\circ}$ increment in range of $\pm 30^{\circ}$, respectively. After calibration of the probe at one chamfer angle, the probe was removed from the calibration device and the chamfer angle was changed by machining the probe in a lathe by using the tool bit at the desired angle.

4.2. Experimental Programme. The chamfer angle of the probe was varied systematically from 30 to $60 \mathrm{deg}$, with gradually increasing chamfer angle at an increment of $5 \mathrm{deg}$.

This range covers most of the usually used chamfer angles. After completing calibration of the probe with a chamfer angle, the probe chamfer angle was changed by machining the probe in a lathe. The accuracy of the probe chamfer angle is checked in Metrology Laboratory, Department of Mechanical engineering, IIT Madras and was found to be within an accuracy of $\pm 0.2 \mathrm{deg}$.

\section{Results and Discussion}

5.1. Sample Data. The pressures measured by the five tubes of the five-hole probe with a chamfer angle of $30^{\circ}$ are presented in Figure 6. The pressures are nondimensionalised with the total pressure and are plotted against the pitch angle. The static pressure measured on the wall of the settling chamber was taken as the total pressure as the magnitude of the dynamic pressure in the settling chamber was very small.

For the sake of clarity, only pressures at three values of yaw angles, namely, $-30^{\circ}, 0^{\circ}$, and, $30^{\circ}$ are presented. The data are presented to see that the data is following the expected trends. Such data for all chamfer angles are plotted to validate the data. However the data are not presented for the sake of brevity.

As expected pressures measured by the central, left, and right holes show parabolic variation with the pitch angle. At $\alpha=-30^{\circ}, P_{L}$ is always more than $P_{C}$, but $P_{R}$ always shows the lowest pressure, as this hole is inclined at a large angle to the flow. At $\alpha=30^{\circ}, P_{R}$ is always more than $P_{C}$, but $P_{L}$ always shows the lowest pressure, as this hole is inclined at a large angle to the flow. At $\alpha=0^{\circ}$, pressures measured by the left and right holes are almost equal at all the pitch angles. Similarly at $\alpha=-30^{\circ}$ and $30^{\circ}$, pressures measured by the top hole are almost equal at all the pitch angles and these pressures are lower than the corresponding pressures at $\alpha=0^{\circ}$. Similar observation is made for pressures measured by the bottom

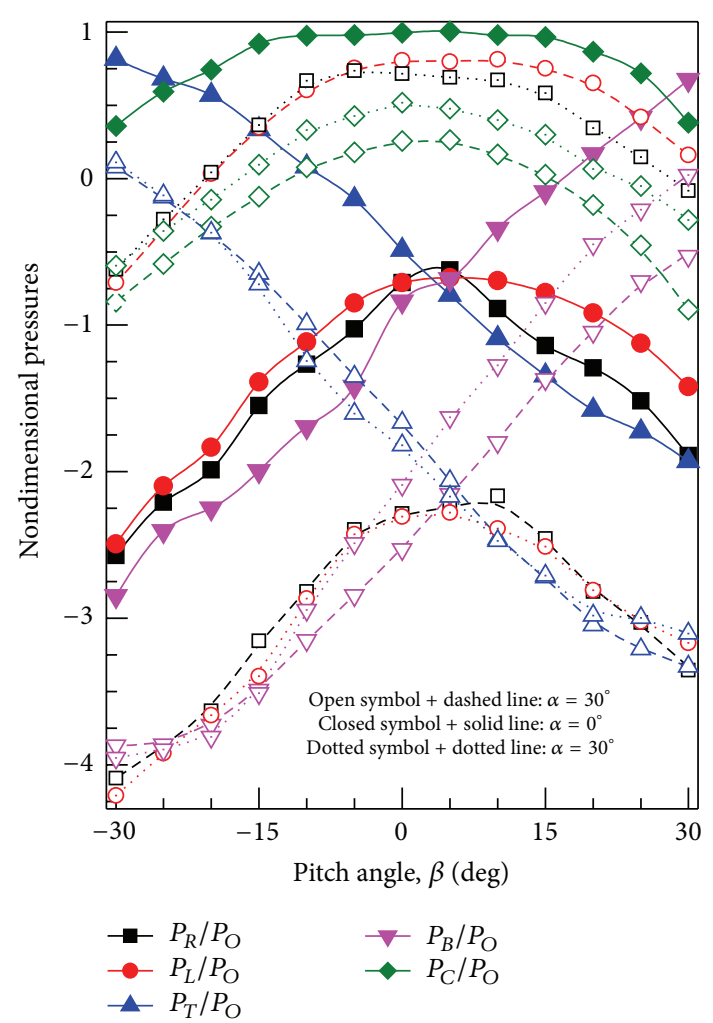

Figure 6: Nondimensional probe pressures for chamfer angle of $30^{\circ}$.

hole. Similar observations are made for the probe heads with different chamfer angles, confirming that the chamfer angle is nearly the same for the side pressure tubes.

5.2. Calibration Coefficients of Five-Hole Probe. The pressures measured by the five holes, the calibration tunnel settling chamber pressure, and calibration section wall static pressure at different values of yaw and pitch angle are used to define nondimensional calibration coefficient as follows:

$$
\begin{gathered}
\underline{P}=\frac{\left(P_{B}+P_{T}+P_{L}+P_{R}\right)}{4}, \\
D=P_{C}-\underline{P}, \\
C_{P \text { YAW }}=\frac{\left(P_{L}-P_{R}\right)}{D}, \\
C_{P \text { PITCH }}=\frac{\left(P_{T}-P_{B}\right)}{D}, \\
C_{P \text { TOTAL }}=\frac{\left(P_{O}-P_{C}\right)}{D}, \\
C_{P \text { STATIC }}=\frac{\left(P_{S}-\underline{P}\right)}{D} .
\end{gathered}
$$

5.3. Calibration Curves of Five-Hole Probe. The calibration curves are presented as follows for the probe with different chamfer angles: 
TABLE 1: Minimum and maximum values and sensitivity of $C_{P Y A W}$.

\begin{tabular}{lcccc}
\hline Chamfer angle & $30^{\circ}$ & $40^{\circ}$ & $50^{\circ}$ & $60^{\circ}$ \\
\hline Minimum value & -4.050 & -6.00 & -16.200 & -7.500 \\
Normalized value & 1.000 & 1.481 & 4.000 & 6.200 \\
Maximum value & 2.380 & 3.000 & 2.605 & 1.852 \\
Normalized value & 1.000 & 1.261 & 22.400 & 3.496 \\
Total difference & 6.430 & 9.00 & 3.484 & 0.293 \\
Normalized value & 1.000 & 1.400 & 3.530 & 0.293 \\
Sensitivity at $\beta=-30^{\circ}$ & 0.083 & 0.140 & 0.121 & 3.530 \\
Normalized value & 1.000 & 1.687 & 2.086 & 0.107 \\
Sensitivity at $\beta=0^{\circ}$ & 0.058 & 0.083 & 0.362 & 3.845 \\
Normalized value & 1.000 & 0.134 & 3.481 \\
Sensitivity at $\beta=30^{\circ}$ & 0.104 & 1.288 & 0.021 \\
Normalized value & 1.000 & & 0.202 \\
\hline
\end{tabular}

TABle 2: Minimum and maximum values and sensitivity of $C_{P \text { PITCH }}$.

\begin{tabular}{lcccc}
\hline Chamfer angle & $30^{\circ}$ & $40^{\circ}$ & $50^{\circ}$ & -12.100 \\
\hline Minimum value & -3.220 & -5.800 & 3.758 & -5.200 \\
Normalized value & 1.000 & 1.801 & 15.000 & 8.615 \\
Maximum value & 3.020 & 5.400 & 4.967 & 2.000 \\
Normalized value & 1.000 & 1.788 & 27.100 & 4.343 \\
Total difference & 6.240 & 11.200 & 0.211 & 2.115 \\
Normalized value & 1.000 & 1.795 & 2.605 & 0.140 \\
Sensitivity at $\alpha=-30^{\circ}$ & 0.081 & 0.108 & 0.090 & 1.728 \\
Normalized value & 1.000 & 1.333 & 1.579 & 0.438 \\
Sensitivity at $\alpha=0 \circ$ & 0.057 & 0.077 & 1.351 & 0.099 \\
Normalized value & 1.000 & 0.179 & 1.737 \\
Sensitivity at $\alpha=30^{\circ}$ & 0.107 & 1.673 & 0.120 \\
Normalized value & 1.000 & & 1.093 \\
\hline
\end{tabular}

(1) $C_{P \text { YAW }}$ versus $C_{P \text { PITCH }}$ for various values of pitch and yaw angles,

(2) $C_{P \text { TOTAL }}$ versus pitch angle for various yaw angles,

(3) $C_{\text {PSTATIC }}$ versus pitch angle for various yaw angles.

These calibration curves are presented and discussed in the following sections.

5.4. Effect of Chamfer Angle on $C_{P Y A W}$ versus $C_{P P I T C H}$ Calibration Curve of Five Hole Probe. The $C_{P Y A W}$ versus $C_{P \mathrm{PITCH}}$ calibration curves of the five hole probes with different chamfer angles are presented in Figure 7 . The corresponding values of yaw and pitch angles are also shown in the figures. All figures are drawn to the same scale. For the sake of clarity only calibration curves at alternate values of yaw angles are shown. The minimum and maximum values of $C_{P Y A W}$ and $C_{P \text { PITCH }}$ for different chamfer angles are presented in Tables 1 and 2 , respectively. Also the sensitivity values at three yaw and pitch angles, namely, $-30,0$, and $30 \mathrm{deg}$, are given. In addition, normalized values of all parameters with respect to the values for the probe with a chamfer angle of $30^{\circ}$ are given in Tables 1 and 2.

From Figure 7 and Tables 1 and 2, it is evident that both $C_{P \text { YAW }}$ and $C_{P \text { PITCH }}$ increase as the probe chamfer angle increases. As the chamfer angle increases, one of the side tubes measure pressure higher than that of the centre tube at some of the yaw and pitch angles. Hence the value of $D$ becomes smaller, causing the values of $C_{P \text { YAW }}$ and $C_{P \text { PITCH }}$ to increase rapidly. In fact the value of $D$ can become negative at large values of yaw and pitch angles, limiting the useful range of the probe. For lower values of probe chamfer angles, the values of $C_{P \text { YAW }}$ and $C_{P \text { PITCH }}$ are comparatively lower. Hence the useful range of yaw and pitch angles for probes with smaller chamfer angle can be more than that for probes with larger chamfer angle. The difference between the minimum and maximum values is also shown in Tables 1 and 2. Again the same trend is observed. As the chamfer angle increases, the side holes sense less of total pressure and more of static pressure. Hence the pressures measured by the side holes will be lesser as the chamfer angle increases. This is a desirable trend. As can be seen from the calibration curves, the range of $C_{P \text { YAW }}$ and $C_{P \text { PITCH }}$ curves increases as the chamfer angle increases. Hence the change in $C_{P Y A W}$ and $C_{P \text { PITCH }}$ is higher as the chamfer angle increases. Hence the sensitivity of $C_{P Y A W}$ and $C_{P \text { PITCH }}$ with yaw and pitch angle increases, giving more accurate interpolated values of yaw and pitch angles as the chamfer angle increases. However this trend is broken when chamfer angle is $55 \mathrm{deg}$. For this and higher chamfer angles, 

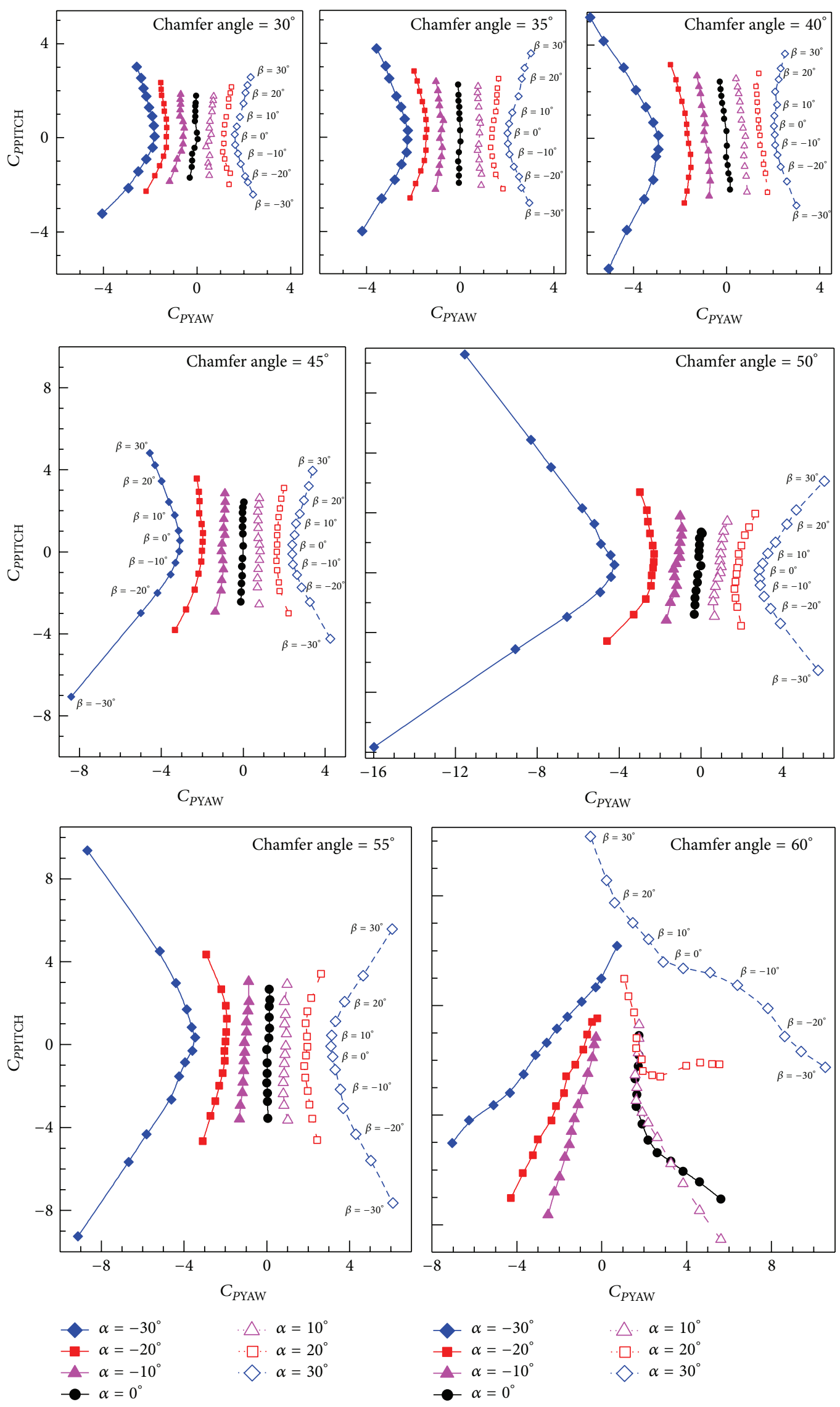

$\triangle \cdot \alpha=10^{\circ}$
$\square \cdot \alpha=20^{\circ}$
$\square \cdot \alpha=30^{\circ}$

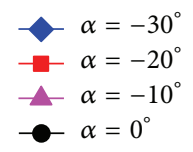

$\triangle . \alpha=10^{\circ}$

$\square . \alpha=20^{\circ}$

$\diamond \cdot \alpha=30^{\circ}$

Figure 7: Calibration curves: $C_{P \text { PITCH }}$ versus $C_{P Y A W}$. 
TABLE 3: Minimum and maximum values and sensitivity of $C_{P \mathrm{TOTAL}}$.

\begin{tabular}{|c|c|c|c|c|}
\hline Chamfer angle & $30^{\circ}$ & $40^{\circ}$ & $50^{\circ}$ & $60^{\circ}$ \\
\hline Minimum value & 0.000 & 0.000 & 0.000 & 0.000 \\
\hline Maximum value & 2.180 & 3.500 & 10.000 & 6.000 \\
\hline Normalized value & 1.000 & 1.606 & 4.587 & 2.752 \\
\hline Total difference & 2.180 & 3.500 & 10.000 & 6.000 \\
\hline Normalized value & 1.000 & 1.606 & 4.587 & 2.752 \\
\hline Sensitivity at $\beta=-30^{\circ}$ & 0.025 & 0.097 & 0.301 & 0.162 \\
\hline Normalized value & 1.000 & 3.880 & 12.040 & 6.480 \\
\hline Sensitivity at $\beta=0^{\circ}$ & 0.014 & 0.030 & 0.052 & 0.080 \\
\hline Normalized value & 1.000 & 2.143 & 3.714 & 5.714 \\
\hline Sensitivity at $\beta=30^{\circ}$ & 0.058 & 0.095 & 0.321 & 0.141 \\
\hline Normalized value & 1.000 & 1.638 & 5.534 & 2.431 \\
\hline Sensitivity at $\alpha=-30^{\circ}$ & 0.037 & 0.040 & 0.104 & 0.111 \\
\hline Normalized value & 1.000 & 1.081 & 2.811 & 3.000 \\
\hline Sensitivity at $\alpha=0^{\circ}$ & 0.015 & 0.019 & 0.023 & 0.029 \\
\hline Normalized value & 1.000 & 1.267 & 1.533 & 1.933 \\
\hline Sensitivity at $\alpha=30^{\circ}$ & 0.059 & 0.086 & 0.289 & 0.074 \\
\hline Normalized value & 1.000 & 1.458 & 4.898 & 1.254 \\
\hline
\end{tabular}

the side holes are more parallel to the streamlines. Hence they sense more static pressure. Also the variation of the side hole pressures may not be much as the yaw and pitch angles increase beyond a certain range. Examination of raw pressure data for probes with these chamfer angles shows that one of the side holes is showing flow separation even when the yaw and pitch angles are low. It is quite likely that different definitions of yaw and pitch coefficients are needed for these chamfer angles. Or zonal method developed by Sitaram and Govardhan [11] may be used. This zonal method is similar to the zonal method used for seven hole probes Venkateswara Babu et al. [12] and Everett et al. [13]. The calibration space is divided into five zones. In each of the zones, one of the five pressures is maximum and this pressure is taken as the representative total pressure. Calibration coefficients are defined differently in each of the zones. The zones are extended so that no calibration space is left without calibration coefficients. The measured pressures are examined to find out the maximum pressure and the calibration curves corresponding to this zone are used to determine the four unknowns, namely, static and total pressures and flow angles. Alternatively the definition of $D$ may be changed following Pisasale and Ahmed [8], Pissasale and Ahmed [14], and Pisasale and Ahmed [15].

5.5. Effect of Chamfer Angle on $C_{\text {PTOTAL versus Pitch Angle }}$ Calibration Curve of Five-Hole Probe. The $C_{P \text { TOTAL versus }}$ pitch angle calibration curves of the five-hole probe with different chamfer angles are presented in Figure 8. These calibration curves are drawn for constant yaw angles. For the sake of clarity only calibration curves at alternate values of yaw angles are shown. All figures are drawn to the same scale. The minimum and maximum values of $C_{P \text { TOTAL }}$ for different chamfer angles are presented in Table 3. Also the sensitivity values at three yaw and pitch angles are given. In addition,

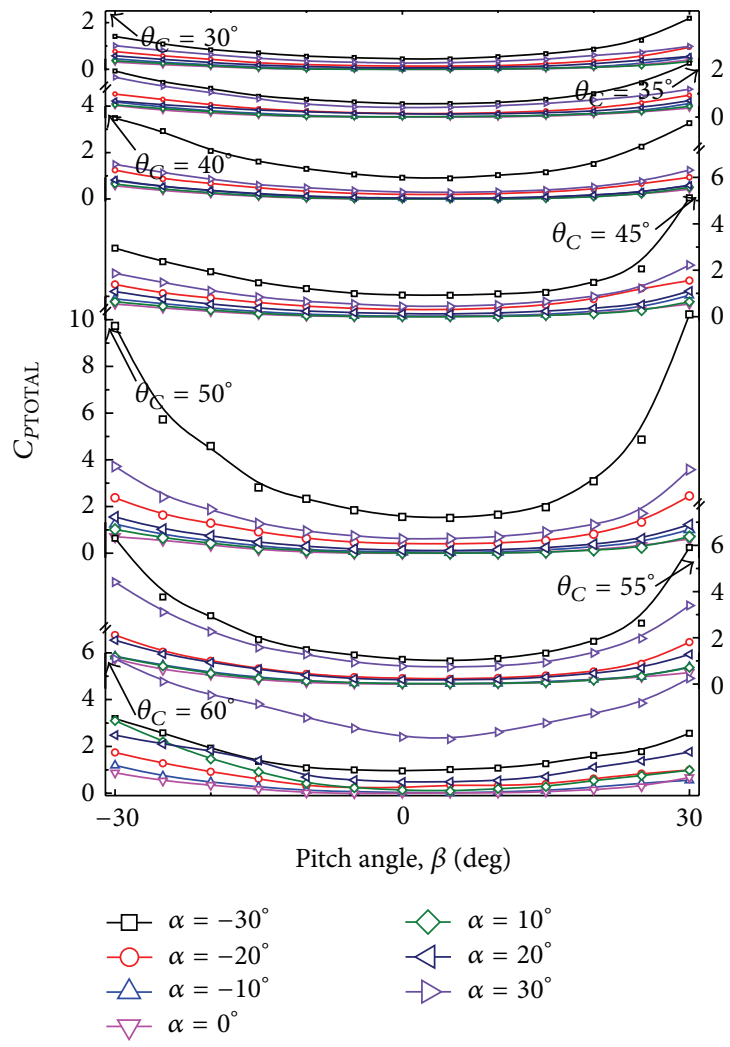

FIGURE 8: Calibration curves: $C_{P \text { TOTAL }}$ versus $\beta$.

normalized values of all parameters with respect to the values for the probe with a chamfer angle of $30^{\circ}$ are given in Table 4 . The minimum value of $C_{P \text { TOTAL }}$ is close to zero, indicating that the central hole is measuring total pressure when the yaw and pitch angles are close to zero. 
Table 4: Minimum and maximum values and sensitivity of $C_{P S T A T I C}$.

\begin{tabular}{|c|c|c|c|c|}
\hline Chamfer angle & $30^{\circ}$ & $40^{\circ}$ & $50^{\circ}$ & $60^{\circ}$ \\
\hline Minimum value & -0.620 & -0.900 & -1.500 & -2.800 \\
\hline Normalized value & 1.000 & 1.452 & 2.419 & 4.516 \\
\hline Maximum value & 0.480 & 0.700 & 0.900 & 0.500 \\
\hline Normalized value & 1.000 & 1.458 & 1.875 & 1.042 \\
\hline Total difference & 1.100 & 1.600 & 2.400 & 3.300 \\
\hline Normalized value & 1.000 & 1.455 & 2.182 & 3.000 \\
\hline Sensitivity at $\beta=-30^{\circ}$ & 0.017 & 0.025 & 0.033 & 0.043 \\
\hline Normalized value & 1.000 & 1.471 & 1.941 & 2.529 \\
\hline Sensitivity at $\beta=0^{\circ}$ & 0.018 & 0.018 & 0.03 & 0.011 \\
\hline Normalized value & 1.000 & 1.000 & 1.667 & 0.611 \\
\hline Sensitivity at $\beta=30^{\circ}$ & 0.018 & 0.019 & 0.008 & 0.003 \\
\hline Normalized value & 1.000 & 1.056 & 0.444 & 0.167 \\
\hline Sensitivity at $\alpha=-30^{\circ}$ & 0.016 & 0.017 & 0.022 & 0.062 \\
\hline Normalized value & 1.000 & 1.063 & 1.375 & 3.875 \\
\hline Sensitivity at $\alpha=0^{\circ}$ & 0.016 & 0.023 & 0.041 & 0.031 \\
\hline Normalized value & 1.000 & 1.438 & 2.563 & 1.938 \\
\hline Sensitivity at $\alpha=30^{\circ}$ & 0.019 & 0.031 & 0.050 & 0.047 \\
\hline Normalized value & 1.000 & 1.632 & 2.632 & 2.474 \\
\hline
\end{tabular}

From Figure 8 and Table 3 , it is evident that $C_{P \text { TOTAL }}$ increases as the probe chamfer angle increases. The difference between the minimum and maximum values is also shown in Table 3. Again the same trend is observed. The central hole always senses more or less the same pressure for the probes with different chamfer angles. However it is normalized with the dynamic pressure based on the probe pressures. As this value gets reduced the value of $C_{P \text { TOTAL increases as the }}$ chamfer angle increases. As the chamfer angle increases, the side holes sense less of total pressure and more of static pressure. Hence the pressures measured by the side holes will be lesser as the chamfer angle increases. This is a desirable trend. As can be seen from the calibration curves, the range of $C_{P \text { TOTAL }}$ curves increases as the chamfer angle increases. Hence the change in $C_{P \text { TOTAL }}$ is higher as the chamfer angle increases. Hence the sensitivity of $C_{P \text { TOTAL }}$ curves with yaw and pitch angle increases, giving more accurate interpolated values of total pressure as chamfer angle increases.

However this trend is broken when chamfer angle is $55 \mathrm{deg}$. For this and higher chamfer angles, the side holes are more parallel to the streamlines. Hence they sense more static pressure. Also the variation of the side hole pressures may not be much as the yaw and pitch angles increase beyond a certain range. It is quite likely that different definition of $C_{P \text { TOTAL }}$ is needed for these chamfer angles. Or alternate techniques described in Section 5.4 may be employed.

5.6. Effect of Chamfer Angle on $C_{\text {PSTATIC versus Pitch Angle }}$ Calibration Curve of Five-Hole Probe. The $C_{P S T A T I C}$ versus pitch angle calibration curves of the five-hole probe with different chamfer angles are presented in Figure 9. These calibration curves are drawn for constant yaw angles. For the sake of clarity only calibration curves at alternate values of yaw angles are shown. All figures are drawn to the same scale. The minimum and maximum values of $C_{P S T A T I C}$ for different chamfer angles are presented in Table 4. Also the sensitivity values at three yaw and pitch angles are given. In addition, normalized values of all parameters with respect to the values for the probe with a chamfer angle of $30^{\circ}$ are given in Table 3 .

From Figure 9 and Table 4 , it is evident that $C_{P S T A T I C}$ increases as the probe chamfer angle increases. The difference between the minimum and maximum values is also shown in Table 4. Again the same trend is observed. The central hole always senses more or less the same pressure for the probes with different chamfer angles. However it is normalized with the dynamic pressure based on the probe pressures. The value of $C_{\text {PSTATIC }}$ depends on the average value of side hole pressures which occurs both in the numerator and denominator. As the chamfer angle increases, the side holes sense less of total pressure and more of static pressure. Hence the pressures measured by the side holes will be lesser as the chamfer angle increases. This is a desirable trend. As can be seen from the calibration curves, the range of $C_{P S T A T I C}$ curves increases as the chamfer angle increases. Hence the change in $C_{P S T A T I C}$ is higher as the chamfer angle increases. Hence the sensitivity of $C_{P S T A T I C}$ curves with yaw and pitch angle increases, giving more accurate interpolated values of static pressures as chamfer angle increases. However this trend is broken when chamfer angle is $55 \mathrm{deg}$. For this and higher chamfer angles, the side holes are more parallel to the streamlines. Hence they sense more static pressure. Also the variation of the side hole pressures may not be much as the yaw and pitch angles increase beyond a certain range. It is quite likely that different definition of $C_{P S T A T I C}$ is needed for these chamfer angle. Or alternate techniques described in Section 5.4 may be employed. 


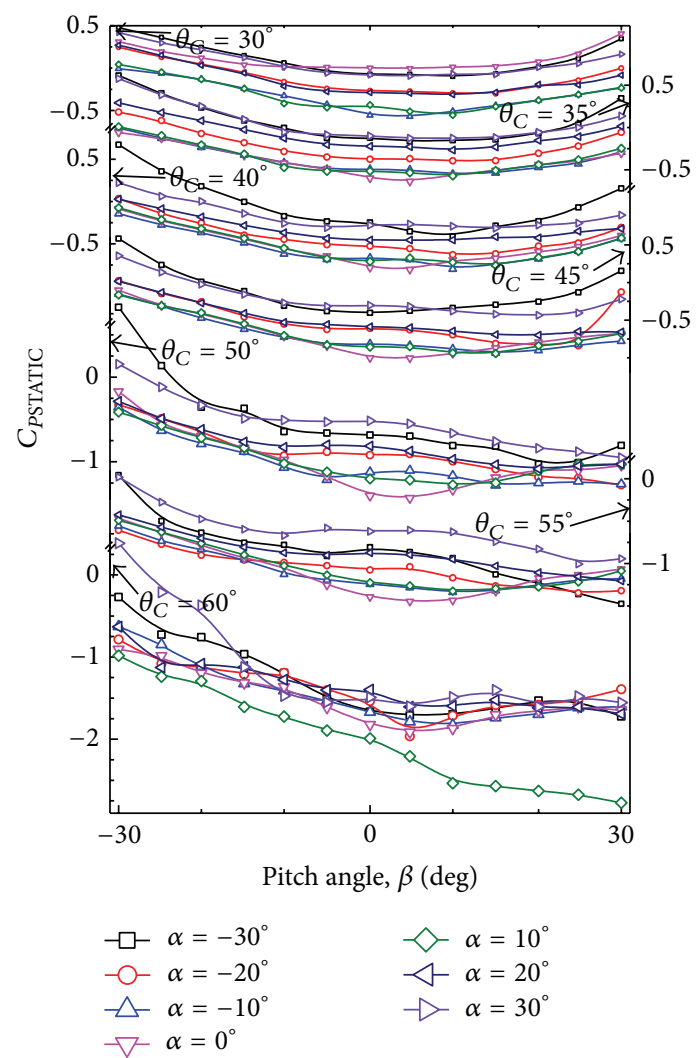

Figure 9: Calibration curves: $C_{\text {PSTATIC }}$ versus $\beta$.

5.7. Effect of Chamfer Angle on Sensitivity of Calibration Coefficients of Five-Hole Probe. The pressure probes used for fluid flow measurements should satisfy two conflicting requirements. They are sensitivity of the probe and operating range of the probe. These conflicting requirements depend on the chamfer angle of the probe head. The present section examines the sensitivity of the probe measurements with yaw and pitch angles. This can be done in two ways: one by examining the sensitivity of the pressures measured by the probe with yaw and pitch angles and the other by examining the sensitivity of the calibration coefficients of the probe with yaw and pitch angles. The second approach is taken in the paper. Ideally the sensitivity has to be determined at all combinations of yaw and pitch angles. While this is possible, it is cumbersome to present and interpret. Hence the sensitivity of various coefficients are examined at three values of yaw and pitch angles, namely, $-30^{\circ}, 0^{\circ}$, and $30^{\circ}$. These values are presented in Tables 1 to 4 . However, for the sake of better visibility, they are plotted in Figure 10. The sensitivities of various coefficients are defined as follows:

$$
C_{P \mathrm{YAW}}: \frac{\left(C_{P \mathrm{YAW}} \text { at } \alpha=30^{\circ}-C_{P \mathrm{YAW}} \text { at } \alpha=-30^{\circ}\right)}{60}
$$

at constant value of pitch angle,

$$
C_{\text {P } \mathrm{PITCH}}: \frac{\left(C_{P \mathrm{PITCH}} \text { at } \beta=30^{\circ}-C_{P \mathrm{PITCH}} \text { at } \beta=-30^{\circ}\right)}{60}
$$

at constant value of yaw angle,

$$
C_{\text {PTOTAL }}: \frac{\left(C_{P \text { TOTAL }} \text { at } \beta= \pm 30^{\circ}-C_{P \text { TOTAL }} \text { at } \beta=0^{\circ}\right)}{30}
$$

at constant value of yaw angle,

$$
C_{\text {PSTATIC }}: \frac{\left(C_{P S T A T I C} \text { at } \beta= \pm 30^{\circ}-C_{P S T A T I C} \text { at } \beta=0^{\circ}\right)}{30}
$$

at constant value of yaw angle,

$$
\begin{array}{r}
C_{\text {PTOTAL }}: \frac{\left(C_{P \text { TOTAL }} \text { at } \alpha= \pm 30^{\circ}-C_{P \text { TOTAL }} \text { at } \alpha=0^{\circ}\right)}{30} \\
\text { at constant value of pitch angle, } \\
C_{P \text { STATIC }}: \frac{\left(C_{P \text { STATIC }} \text { at } \alpha= \pm 30^{\circ}-C_{P S T A T I C} \text { at } \alpha=0^{\circ}\right)}{30}
\end{array}
$$

at constant value of pitch angle.

Larger value of sensitivity of $C_{P \text { TOTAL }}$ and $C_{P S T A T I C}$ is used at yaw and pitch angles of $-30^{\circ}$ or $30^{\circ}$.

From Figure 10, it can be seen that the sensitivity of $C_{P \text { YAW }}, C_{P \text { PITCH }}$, and $C_{P \text { TOTAL }}$ gradually increases with probe chamfer angle and the maximum value occurs for the probe with a chamfer angle of $50^{\circ}$. As the chamfer angle further increases, the sensitivity of these coefficients decreases. The static pressure coefficient has very low value of sensitivity and its value is nearly independent of probe chamfer angle.

\section{Conclusions}

The following major conclusions are drawn from the present experimental investigation on the effect of chamfer angle on the calibration curves of five hole probes.

(1) The value of the calibration coefficients increases as the chamfer angle increases. This trend is observable up to the chamfer angle of $50 \mathrm{deg}$. For this chamfer angle, the calibration coefficients show maximum values. This is a desirable trend, as the sensitivity of the calibration coefficients increases, more accurate values of interpolated values of yaw and pitch angles and total and static pressures can be obtained. However the useful range of the probe is limited to about $30^{\circ}$.

(2) If the flow to be measured is expected to have large changes in yaw and pitch angles, probe with a chamfer angle of about $30^{\circ}$ is desirable. Probe with this chamfer angle has large useful range.

(3) Sensitivity of static pressure coefficient is nearly independent of probe chamfer angle.

(4) For the chamfer angles of $55 \mathrm{deg}$. and higher values, alternate techniques given at the end of Section 5.4 may be used. 

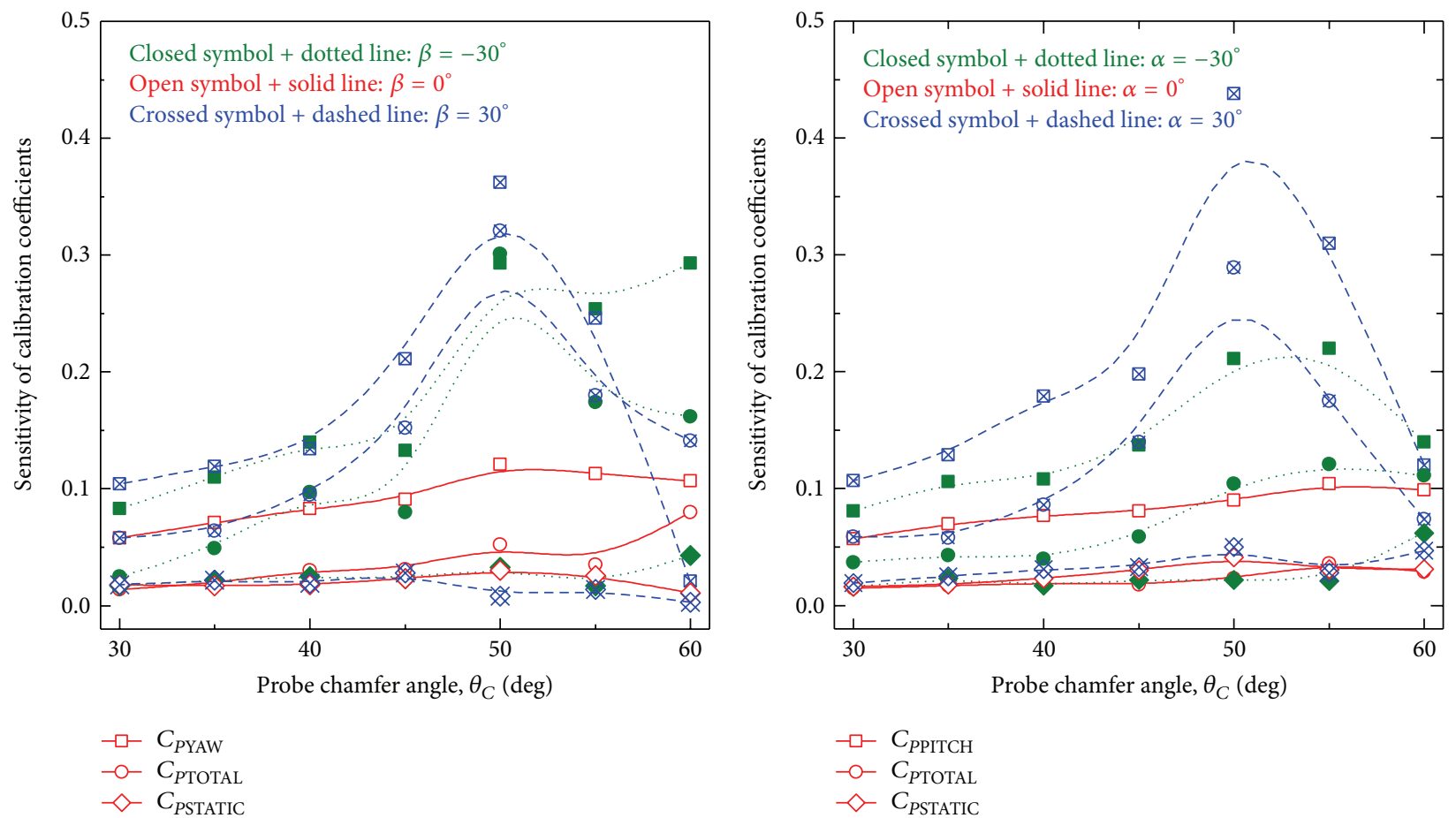

FIGURE 10: Sensitivity of calibration coefficients.

\section{Nomenclature}

$\begin{array}{ll}C_{P \text { PITCH }}: & \text { Pitch coefficient (defined in text) } \\ C_{P S T A T I C}: & \text { Static pressure coefficient (defined in } \\ & \text { text) } \\ C_{P Y A W}: & \text { Yaw coefficient (defined in text) } \\ C_{P \text { TOTAL }}: & \text { Total pressure coefficient (defined in text) } \\ D: & \text { Probe dynamic head (Pa) (defined in } \\ & \text { text) } \\ P_{P}: P_{C}, P_{L}, P_{R}, P_{T}: & \text { Pressures measured by bottom, centre, } \\ & \text { left, right and top holes of the probe } \\ & \text { (please see Figure 2 for identification of } \\ & \text { holes) } \\ P_{O}: & \text { Total pressure (Pa) } \\ P_{S}: & \text { Static pressure (Pa) } \\ \alpha: & \text { Yaw Angle (deg.) } \\ \beta: & \text { Pitch Angle (deg.) } \\ \theta_{C}: & \text { Chamfer Angle (deg.). }\end{array}$

\section{Conflict of Interests}

The authors declare that there is no conflict of interests regarding the publication of this paper.

\section{Acknowledgments}

The authors would like to thank Mr. P. Perumal of Thermal Turbomachines Laboratory for fabricating the probe and other technical and administrative staff of Thermal Turbomachines Laboratory, Department of Mechanical Engineering,
IIT Madras, for their help. The authors would like to thank the reviewers for their suggestions, which improved the quality of the paper substantially.

\section{References}

[1] A. L. Treaster and A. M. Yocum, "The calibration and application of five-hole probes," ISA Transactions, vol. 18, no. 3, pp. 2334, 1979.

[2] V. Malviya, R. Mishra, and E. Palmer, "CFD investigation on 3dimensional interference of a five-hole probe in an automotive wheel arch," Advances in Mechanical Engineering, vol. 2010, Article ID 763718, 19 pages, 2010.

[3] J. W. Naughton, L. N. Cattafesta III, and G. S. Settles, "Miniature, fast-response five-hole conical probe for supersonic flowfield measurements," AIAA Journal, vol. 31, no. 3, pp. 453-458, 1993.

[4] I. M. Milanovic and I. M. Kalkhoran, "Numerical calibration of a conical five-hole probe for supersonic measurements," Measurement Science and Technology, vol. 11, no. 12, pp. 1812$1818,2000$.

[5] S. J. Lien and N. A. Ahmed, "An examination of suitability of multi-hole pressure probe technique for skin friction measurement in turbulent flow," Flow Measurement and Instrumentation, vol. 22, no. 3, pp. 215-224, 2011.

[6] D. Telionis, Y. Yang, and O. Rediniotis, "Recent developments in multi-hole probe technology," in Proceedings of the 20th International Congress of Mechanical Engineering (COBEM '09), Gramado, Brazil, November 2009.

[7] P. M. Ligrani, B. A. Singer, and L. R. Baun, "Miniature fivehole pressure probe for measurement of three mean velocity components in low-speed flows," Journal of Physics E: Scientific Instruments, vol. 22, no. 10, pp. 868-876, 1989. 
[8] A. J. Pisasale and N. A. Ahmed, "A novel method for extending the calibration range of five-hole probe for highly threedimensional flows," Flow Measurement and Instrumentation, vol. 13, no. 1-2, pp. 23-30, 2002.

[9] K. M. A. Díaz, J. M. F. Oro, and E. B. Marigorta, "Direct calibration framework of triple-hole pressure probes for incompressible flow," Measurement Science and Technology, vol. 19, no. 7, Article ID 075401, 2008.

[10] R. G. Dominy and H. P. Hodson, "An investigation of factors influencing the calibration of five-hole probes for threedimensional flow measurements," Journal of Turbomachinery, vol. 115, no. 3, pp. 513-519, 1993.

[11] N. Sitaram and M. Govardhan, "Large angle calibration of five hole probes," Journal of the Aeronautical Society of India, vol. 54, no. 3, pp. 265-272, 2002.

[12] C. Venkateswara Babu, M. Govardhan, and N. Sitaram, "A method of calibration of a seven-hole pressure probe for measuring highly three-dimensional flows," Measurement Science and Technology, vol. 9, no. 3, pp. 468-476, 1998.

[13] K. N. Everett, A. A. Gerner, and D. A. Durston, "Seven-hole probe for high angle flow measurement: theory and calibration," AIAA Journal, vol. 21, no. 7, pp. 992-998, 1983.

[14] A. Pissasale and N. A. Ahmed, "Theoretical calibration of a five hole probe for highly three dimensional flow," Measurement Science and Technology, vol. 13, pp. 1100-1107, 2002.

[15] A. J. Pisasale and N. A. Ahmed, "Development of a functional relationship between port pressures and flow properties for the calibration and application of multihole probes to highly threedimensional flows," Experiments in Fluids, vol. 36, no. 3, pp. 422-436, 2004. 

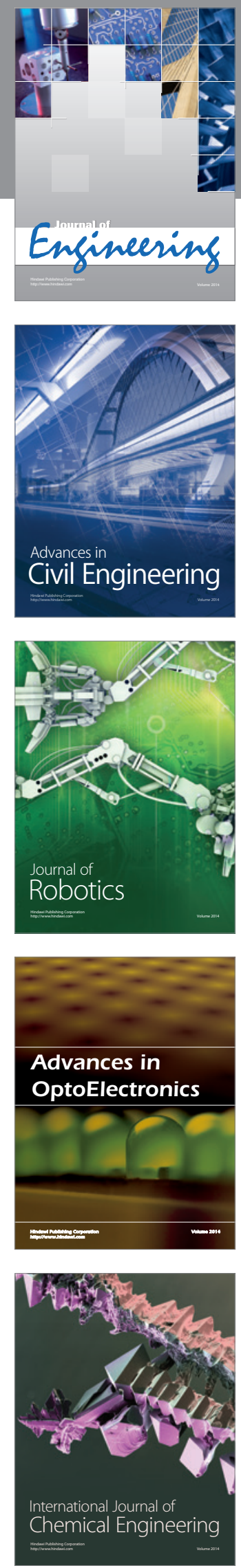

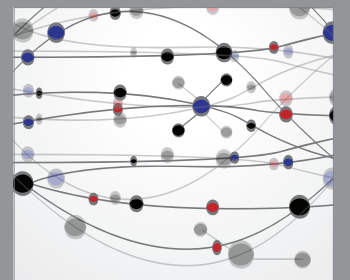

The Scientific World Journal
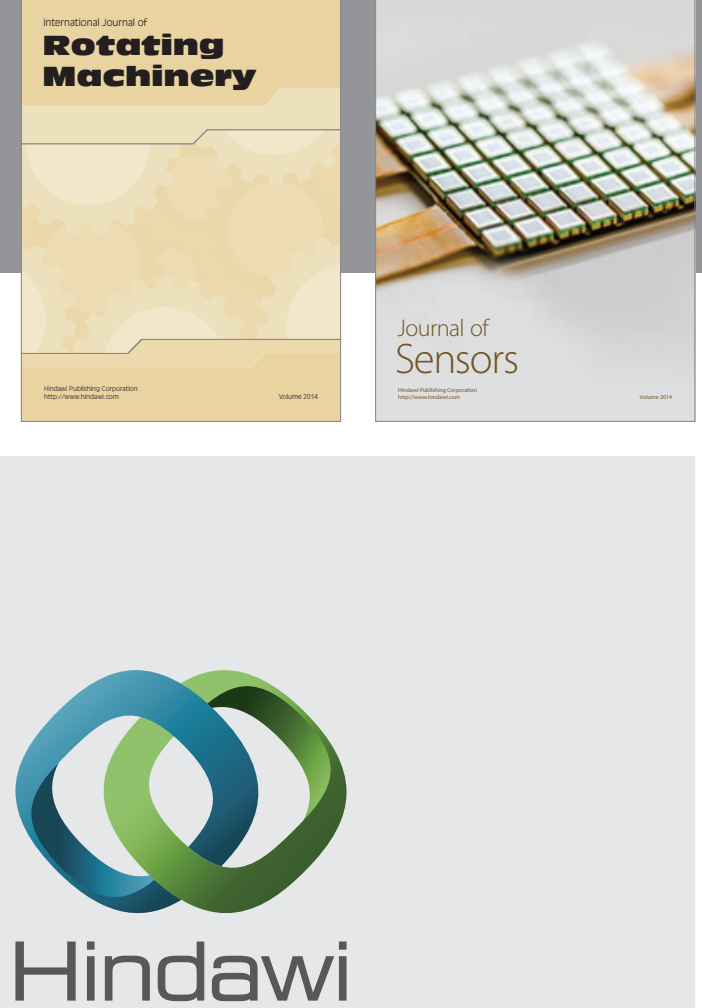

Submit your manuscripts at http://www.hindawi.com
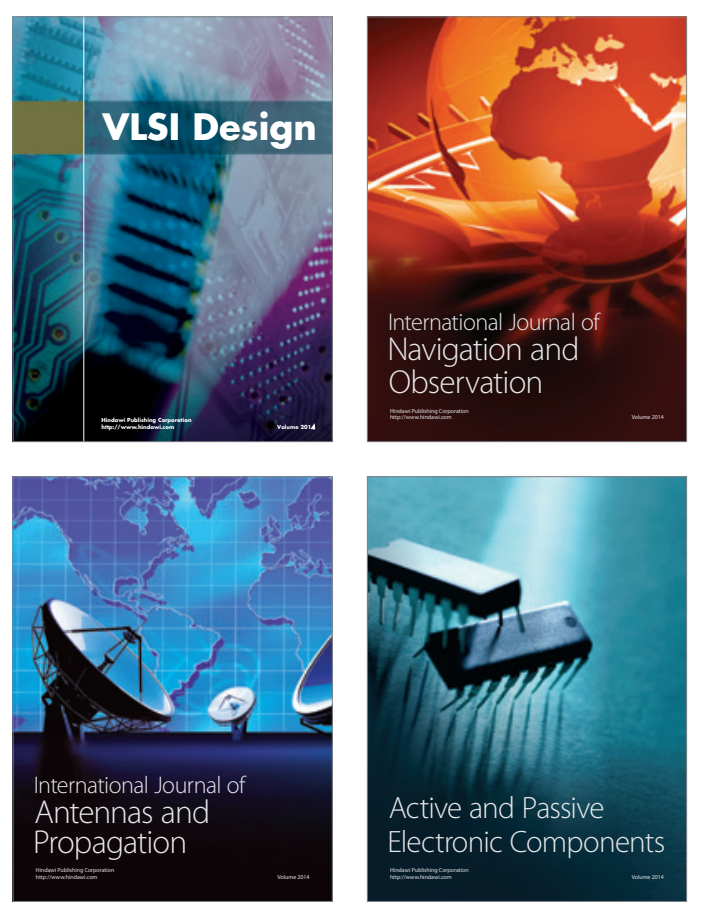
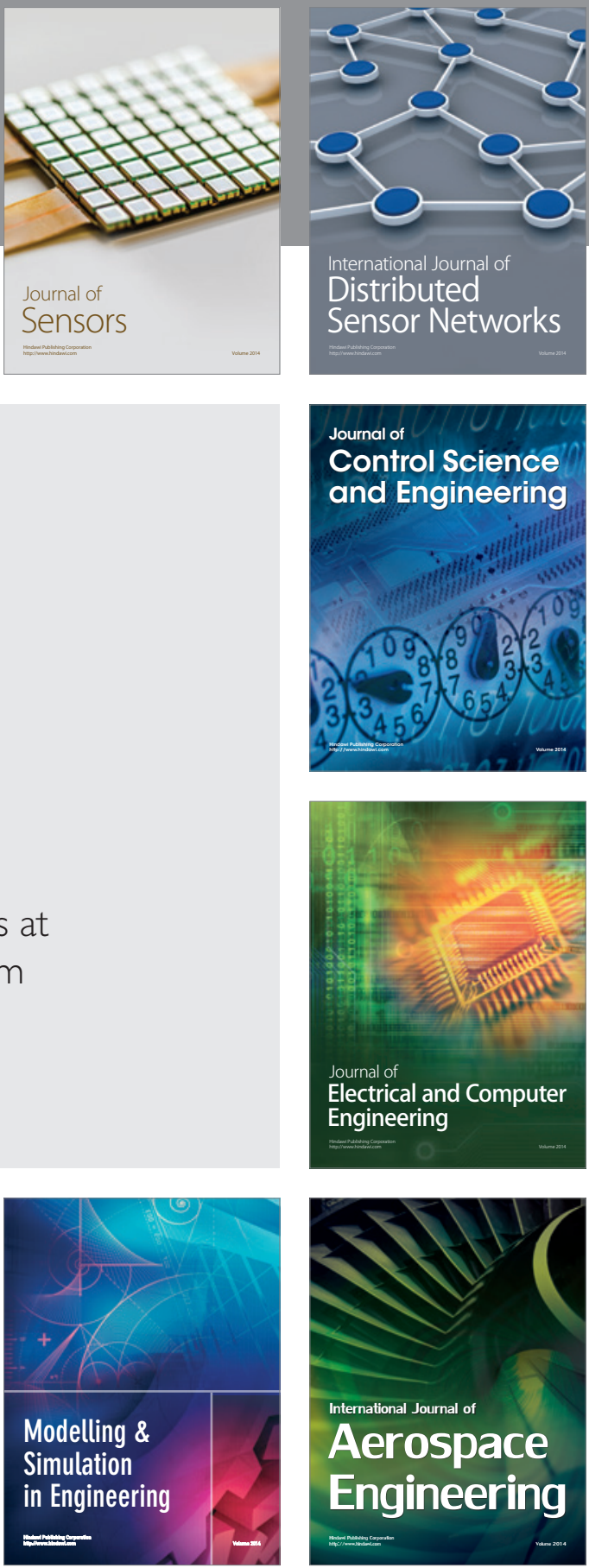

Journal of

Control Science

and Engineering
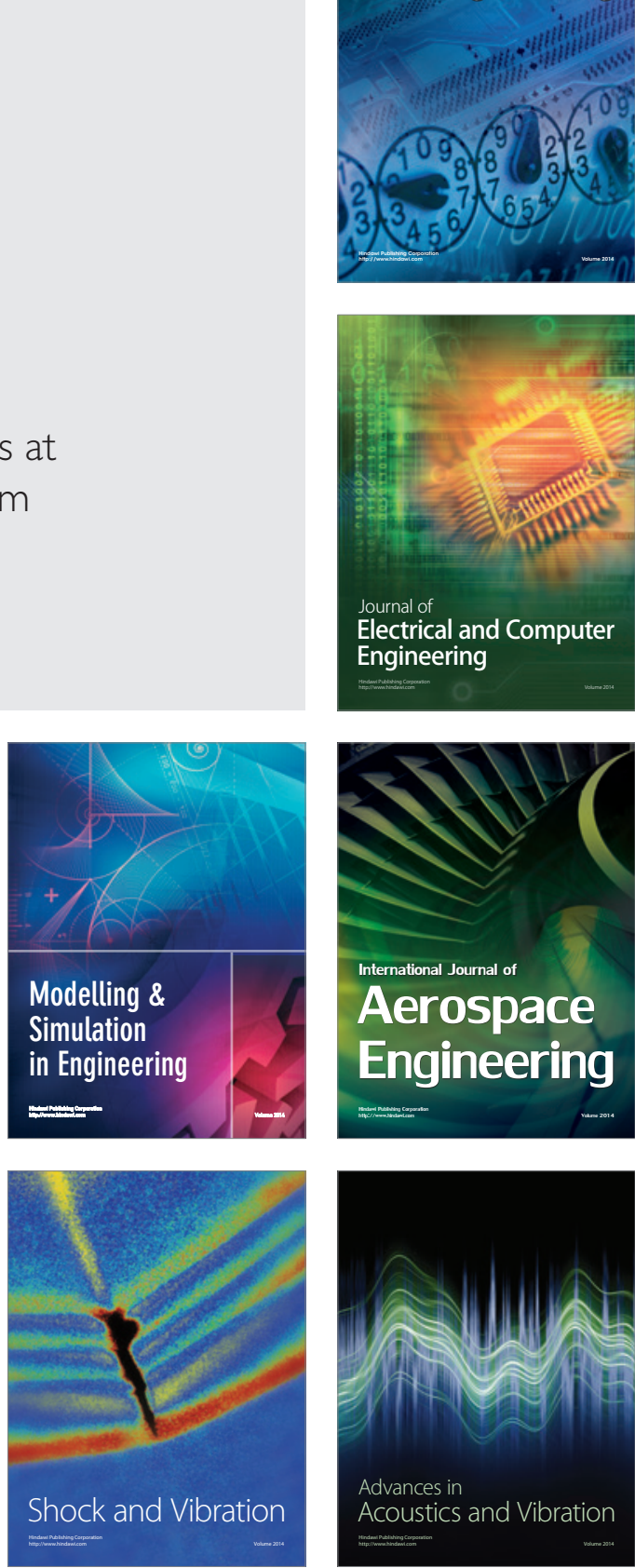